\title{
One-pot synthesis of 5-carboxanilide-dihydropyrimidinones using etidronic Acid
}

\author{
Akshay M. Pansuriya, Mahesh M. Savant, Chirag V. Bhuva, Jyoti Singh, and Yogesh T. \\ Naliapara*
}

Chemical Research Laboratory, Department of Chemistry, Saurashtra University, Rajkot360005, India

E-mail: naliaparachem@yahoo.co.in

\begin{abstract}
A combination of a modified Biginelli reaction and an etidronic acid prompted a cyclocondensation reaction providing 5-carboxanilide-dihydropyrimidinones in good to excellent yields.
\end{abstract}

Keywords: Biginelli reaction, etidronic acid, cyclocondensation, 5-carboxanilidedihydropyrimidinones, excellent yields

\section{Introduction}

Pyrimidines have been subjected to a large number of different modifications in order to obtain derivatives having different biological properties. Several groups have studied the chemistry and pharmacological properties of pyrimidine derivatives. ${ }^{1-14}$ Pyrimidines have been found to have a broad range of biological effects including antiviral, antitumor, antibacterial, anti-inflammatory, ${ }^{1}$ antihypertensive, ${ }^{2}$ cardiovascular, ${ }^{3}$ calcium channel blocking, ${ }^{4}$ and neuropeptide Y (NPY) antagonistic activity. ${ }^{15}$ The versatile biological properties of pyrimidine derivatives prompted us to take up this project to synthesize some novel derivatives using a cyclocondensation reaction of a 1,3-diketone, an aldehyde, and urea.

The synthetic methodology used to generate DHPMs has been well documented and has typically involved variations of the original Biginelli reaction. ${ }^{1,16-18}$ The reports by Kappe and Falsone $^{11-19}$ describe the synthesis of these compounds via a one pot condensation utilizing polyphosphate ester (PPE) ${ }^{19}$ to generate 5-carboxylate DHPMs, however, 5-carboxanilidedihydropyrimidinones using etidronic acid were not exemplified.

Etidronic acid [(1-hydroxyethylidene)bisphosphonic acid] is a phosphonic acid and is also known as a bisphosphonate having a molecular formula $\mathrm{C}_{2} \mathrm{H}_{8} \mathrm{O}_{7} \mathrm{P}_{2}$. The two $\mathrm{PO}_{3}$ (phosphonate) 
groups are covalently linked to a single carbon atom. ${ }^{20}$ It is different from PPE and polyphosphoric acid (PPA).

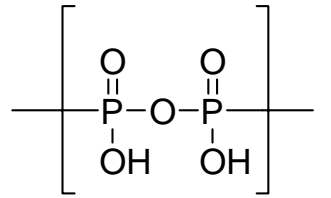

Polyphosphoric acid

$\mathrm{pKa}=0.85$

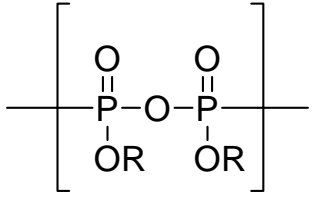

Polyposphate Ester

$\mathrm{pKa}=1.5$

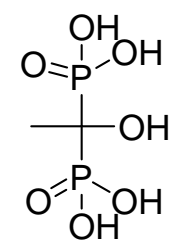

Etidronic acid

$\mathrm{pK}_{1}=1.35$

$\mathrm{pK}_{2}=2.87$

\section{Figure 1}

Reports have shown that polyphosphate ester (PPE), when used in an aprotic solvent such as THF, can serve as a desicant, ${ }^{19}$ and simple benzamides can be dehydrated to their corresponding benzonitriles using this reagent. ${ }^{21}$ Etidronic acid can also serve as a desicant. Our plan was to investigate whether etidronic acid could serve as a suitable reagent to form 5-carbanilideDHPMs. In principle, etidronic acid should be strong enough as desicant to dehydrate a diketone, an aldehyde and urea to give the desired DHPMs, while being mild enough (Figure 1) to allow the use of functionally sensitive aldehydes.

\section{Results and Discussion}

After extensive optimization, we found that the single step reaction could be carried out in a general and efficient one-pot process to afford a variety of 5-carboxanilide-DHPMs. As outlined in Table 1, addition of 0.3 equiv of etidronic acid to a reaction mixture containing a 1:1:1.5 ratio of aldehyde/acetoacetanilide/urea in THF, at $75{ }^{\circ} \mathrm{C}$ for $1 \mathrm{hr}$, was optimal for the formation of 5carboxanilide-DHPMs without affecting the anilide group in yields typically exceeding $70 \%$.

A variety of substituted aromatic, aliphatic and hetero-aromatic aldehydes, with either electron-donating or electron-withdrawing groups, provided favorable results in this reaction. For example, 5-carboxanilide-dihydropyrimidinones generated from 4-nitro benzaldehyde and 2furyl aldehyde afforded the corresponding products in greater than $90 \%$ yield. Aliphatic aldehydes were equally amenable to these conditions with $n$-butyraldehyde providing the 5 carboxanilide-dihydropyrimidinone in 95 to $97 \%$ yield. Halogenated aromatic substitution at the 4-position of the DHPM could also be achieved using this methodology, albeit in significantly lower yields (compound 2, 4 and 7). 


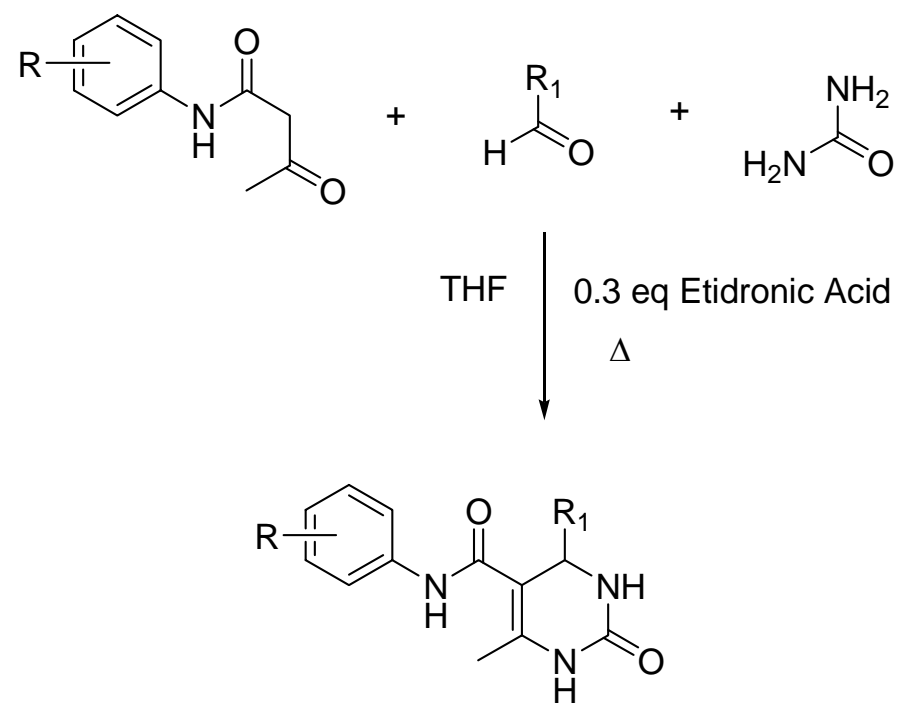

Scheme 1. Sunthetic approach towards 5-carboxanilide-4-substituted dihydropyrimidinones.

All the synthesized compounds were characterized using IR, Mass, ${ }^{1} \mathrm{H}$ NMR, ${ }^{13} \mathrm{C}$ NMR and elemental analysis. In the mass spectral study, the molecular ion peak was observed in agreement with the molecular weight of the respective compound. In the ${ }^{1} \mathrm{H}$ NMR spectral study, two characteristic signals of $-\mathrm{NH}$ of the dihydropyrimidine ring system were observed between 8.5 to $10 \delta \mathrm{ppm}$. The chiral proton at the $\mathrm{C} 4$ position exhibited a signal at $5-5.5 \delta \mathrm{ppm}$ as a singlet. The $-\mathrm{NH}$ proton of the carboxanilide group was observed at $8-8.5 \delta \mathrm{ppm}$ as a singlet. The ${ }^{13} \mathrm{C}$ NMR spectra were consistent with the proposed structure. There are two carbon signals found at 163 to $165 \delta \mathrm{ppm}$ for the amide carbon $(\mathrm{CONH})$. The reason of having more carbon signals than expected could be due to two isomers may existing because of a restricted rotation around the amide functional group. IR spectra showed a secondary amine (-NH) group in the range of 3200 to $3400 \mathrm{~cm}^{-1}$ and a carbonyl $(-\mathrm{C}=\mathrm{O})$ group near $1690 \mathrm{~cm}^{-1}$.

Table 1. A general one-pot Biginelli reaction to generate 5-carboxanilide-4-substituted dihydropyrimidinones

\begin{tabular}{|c|c|c|c|}
\hline Entry & $\mathrm{R}$ & R1 & Yield $^{\mathrm{a}}(\%)$ \\
\hline 1 & $3-\mathrm{CF}_{3}$ & Phenyl & 79 \\
\hline 2 & $3-\mathrm{CF}_{3}$ & 3-Cl Phenyl & 69 \\
\hline 3 & $3-\mathrm{CF}_{3}$ & 2-Furyl & 90 \\
\hline 4 & 3,4-dichloro & 3-Cl Phenyl & 73 \\
\hline 5 & 3,4-dichloro & 4- $\mathrm{NO}_{2}$ Phenyl & 92 \\
\hline 6 & 3,4-dichloro & n-propyl & 97 \\
\hline 7 & $4-\mathrm{NO}_{2}$ & 4-F Phenyl & 72 \\
\hline 8 & $4-\mathrm{NO}_{2}$ & 4- $\mathrm{OCH}_{3}$ Phenyl & 85 \\
\hline 9 & $4-\mathrm{NO}_{2}$ & n-propyl & 95 \\
\hline
\end{tabular}

${ }^{\mathrm{a}}$ Isolated yields after purification. 


\section{Conclusion}

In summary, a new method for the preparation of 5-carboxanilide-DHPMs was discovered that utilizes a multicomponent coupling reaction catalyzed by etidronic acid, with a rapid and high yielding cyclocondensation to afford the corresponding DHPMs. The use of etidronic acid was well tolerated with a range of aldehydes and ketones. In addition, this methodology is cost effective and amenable to large-scale syntheses.

\section{Experimental Section}

General Procedures. Melting points were determined on an electro thermal apparatus using open capillaries and are uncorrected. Thin-layer chromatography was accomplished on 0.2-mm precoated plates of silica gel G60 $\mathrm{F}_{254}$ (Merck). Visualization was made with UV light (254 and $365 \mathrm{~nm}$ ) or with an iodine vapor. IR spectra were recorded on a FTIR-8400 spectrophotometer using DRS prob. ${ }^{1} \mathrm{H}$ NMR spectra were recorded on a Bruker AVANCE II (400 MHz) spectrometer in DMSO. Chemical shifts are expressed in $\delta \mathrm{ppm}$ downfield from TMS as an internal standard. Mass spectra were determined using direct inlet probe on a GCMS-QP 2010 mass spectrometer (Shimadzu). Solvents were evaporated with a BUCHI rotary evaporator. All reagents were purchased from Fluka, Sigma Aldrich, Merck and Rankem and used without further purification.

\section{5-Carboxanilide-tetrahydropyrimidines. General Procedure}

A mixture of an appropriate aldehyde (10 mmol, 1.0 equiv), $N$-phenyl-3-oxobutanamide (10 mmol, 1.0 equiv), urea ( $15 \mathrm{mmol}, 1.5$ equiv) and etidronic acid ( $5.0 \mathrm{mmol}, 0.5$ equiv) in THF (10 $\mathrm{mL}$ ) was heated in a sealed tube for $1 \mathrm{hr}$ at $75{ }^{\circ} \mathrm{C}$. The reaction mixture was cooled to room temperature and poured onto ice $(100 \mathrm{~g})$. The resulting precipitate was collected by vacuum filtration and the solid was washed with water followed by a small amount of methanol and diethyl ether. The solid was recrystallized from ethanol to yield N-phenyl-1,2,3,4-tetrahydro-6methyl-2-oxo-4-phenylpyrimidine-5-carboxamide derivatives as a white to pale yellow solid in $70-95 \%$ yield.

$\mathrm{N}$-(3-(Trifluoromethyl)phenyl)-4-phenyl-1,2,3,4-tetrahydro-6-methyl-2-oxo-pyrimidine-5carboxamide (1). Mp 208-210 ${ }^{\circ} \mathrm{C}$; Yield - 79\%. IR (KBr): 3289, 3276, 3234, 3010, 2956, 1689 , 1672, 1554, 1345, 1220, 1108, $789 \mathrm{~cm}^{-1} ;{ }^{1} \mathrm{H}$ NMR: $\delta=10.17$ (s, 1H, NH), $9.95(\mathrm{~s}, 1 \mathrm{H}, \mathrm{NH}), 8.39$ (s, 1H, NH), 8.09-7.27 (m, 9H, Ar), $5.46(\mathrm{~s}, 1 \mathrm{H}, \mathrm{CH}), 1.61\left(\mathrm{~s}, 3 \mathrm{H}, \mathrm{CH}_{3}\right)$; Mass: $m / z=375\left[\mathrm{M}^{+}\right]$; ${ }^{13} \mathrm{C}$ NMR (400 MHz, DMSO) $\delta 14.13\left(\mathrm{CH}_{3}\right), 51.12(\mathrm{C} 4), 108.73$ (C5), 120.54, 121.16, 125.81, $126.19,127.34,128.50,128.68,130.08,130.31,130.36,132.17$ and 133.00 (CAr.), 125.71 $\left(\mathrm{CF}_{3}\right), 134.08$ (C-C4, Ar), 137.34 (C-NH, Ar), 146.35 (C6), 151.05 (C2, CO), $164.35\left(\mathrm{CONH}_{2}\right)$; Anal. Calcd for $\mathrm{C}_{19} \mathrm{H}_{16} \mathrm{~F}_{3} \mathrm{~N}_{3} \mathrm{O}_{2}: \mathrm{C}, 60.80 ; \mathrm{H}, 4.30 ; \mathrm{N}, 11.20$. Found: $\mathrm{C}, 60.77 ; \mathrm{H}, 4.28 ; \mathrm{N}, 11.21$. 
$N$-(3-(Trifluoromethyl)phenyl)-4-(3-chlorophenyl)-1,2,3,4-tetrahydro-6-methyl-2-

oxopyrimidine-5-carboxamide (2). Mp 216-218 ${ }^{\circ} \mathrm{C}$; Yield - 69\%. IR (KBr): 3408, 3298, 3271, 3088, 2928, 1672, 1654, 1506, 1442, 1334, 1122, $792 \mathrm{~cm}^{-1} ;{ }^{1} \mathrm{H}$ NMR: $\delta=10.14(\mathrm{~s}, 1 \mathrm{H}, \mathrm{NH}), 9.98$ (s, 1H, NH), 8.45 (s, 1H, NH), 8.09-7.22 (m, 8H, Ar), $5.46(\mathrm{~s}, 1 \mathrm{H}, \mathrm{CH}), 1.64$ (s, 3H, $\left.\mathrm{CH}_{3}\right)$; Mass: $m / z=409\left[\mathrm{M}^{+}\right]$; Anal. Calcd for $\mathrm{C}_{19} \mathrm{H}_{15} \mathrm{ClF}_{3} \mathrm{~N}_{3} \mathrm{O}_{2}$ : C, 55.69; H, 3.69; N, 10.25. Found: $\mathrm{C}$, 55.62; H, 3.67; N, 10.22 .

$\mathrm{N}$-(3-(Trifluoromethyl)phenyl)-4-(furan-2-yl)-1,2,3,4-tetrahydro-6-methyl-2-

oxopyrimidine-5-carboxamide (3). Mp 222-224 ${ }^{\circ} \mathrm{C}$; Yield - 90\%. IR (KBr): 3290, 3282, 3271, 3088, 2856, 1690, 1672, 1376, 1222, 1022, $756 \mathrm{~cm}^{-1} ;{ }^{1} \mathrm{H}$ NMR: $\delta=10.04$ (s, 1H, NH), 9.97 (s, 1H, NH), 8.40 (s, 1H, NH), 7.84-6.79 (m, 7H, Ar), 4.96 (s, 1H, CH), 1.73 (s, 3H, $\mathrm{CH}_{3}$ ); Mass: $m / z=365\left[\mathrm{M}^{+}\right]$; Anal. Calcd for $\mathrm{C}_{17} \mathrm{H}_{14} \mathrm{~F}_{3} \mathrm{~N}_{3} \mathrm{O}_{3}: \mathrm{C}, 55.89 ; \mathrm{H}, 3.86 ; \mathrm{N}, 11.50$. Found: C, 55.85; $\mathrm{H}$, $3.84 ; \mathrm{N}, 11.49$.

$N$-(3,4-Dichlorophenyl)-4-(3-chlorophenyl)-1,2,3,4-tetrahydro-6-methyl-2-oxopyrimidine-5carboxamide (4). Mp 192-194 ${ }^{\circ} \mathrm{C}$; Yield - 73\%. IR (KBr): 3450, 3209, 3072, 2847, 1691, 1676, 1600, 1510, 1425, 1342, 1172, 1023, $769 \mathrm{~cm}^{-1} ;{ }^{1} \mathrm{H}$ NMR: $\delta=9.70(\mathrm{~s}, 1 \mathrm{H}, \mathrm{NH}), 8.92(\mathrm{~s}, 1 \mathrm{H}, \mathrm{NH})$, 8.42 (s, 1H, NH), 7.94-7.15 (m, 7H, Ar), $5.00(\mathrm{~s}, 1 \mathrm{H}, \mathrm{CH}), 1.73\left(\mathrm{~s}, 1 \mathrm{H}, \mathrm{CH}_{3}\right) ; \mathrm{Mass}: \mathrm{m} / z=410$ $\left[\mathrm{M}^{+}\right]$; Anal. Calcd for $\mathrm{C}_{18} \mathrm{H}_{14} \mathrm{Cl}_{3} \mathrm{~N}_{3} \mathrm{O}_{2}: \mathrm{C}, 52.64 ; \mathrm{H}, 3.44 ; \mathrm{N}, 10.23$. Found: $\mathrm{C}, 52.61 ; \mathrm{H}, 3.45 ; \mathrm{N}$, 10.21 .

$\mathbf{N}$-(3,4-Dichlorophenyl)-4-(4-nitrophenyl)-1,2,3,4-tetrahydro-6-methyl-2-oxopyrimidine-5carboxamide (5). Mp $186-188^{\circ} \mathrm{C}$; Yield - 92\%. IR (KBr): 3284, 3228, 3117, 2933, 2854, 1693, 1666, 1622, 1442, 1338, 1238, 1128, $773 \mathrm{~cm}^{-1} ;{ }^{1} \mathrm{H}$ NMR: $\delta=10.25$ (s, 1H, NH), 9.85 (s, $1 \mathrm{H}$, $\mathrm{NH}), 8.20-7.24(\mathrm{~m}, 7 \mathrm{H}, \mathrm{Ar}), 5.07(\mathrm{~s}, 1 \mathrm{H}, \mathrm{CH}), 1.62\left(\mathrm{~s}, 3 \mathrm{H}, \mathrm{CH}_{3}\right)$; Mass: $m / z=421\left[\mathrm{M}^{+}\right]$; Anal. Calcd for $\mathrm{C}_{18} \mathrm{H}_{14} \mathrm{Cl}_{2} \mathrm{~N}_{4} \mathrm{O}_{4}$ : C, 51.32; H, 3.35; N, 13.30. Found: C, 51.27; H, 3.31; N, 13.34.

$\mathbf{N}$-(3,4-Dichlorophenyl)-4-propyl-1,2,3,4-tetrahydro-6-methyl-2-oxo-pyrimidine-5carboxamide (6). Mp 184-186 C; Yield - 97\%. IR (KBr): 3286, 3272, 2976, 2842, 1684, 1456, 1361, 1222, 1072, $740 \mathrm{~cm}^{-1}$; ${ }^{1} \mathrm{H}$ NMR: $\delta=10.44(\mathrm{~s}, 1 \mathrm{H}, \mathrm{NH}), 9.57(\mathrm{~s}, 1 \mathrm{H}, \mathrm{NH}), 7.60-7.19(\mathrm{~m}$, $3 \mathrm{H}, \mathrm{Ar}), 6.87(\mathrm{~s}, 1 \mathrm{H}, \mathrm{NH}), 4.95(\mathrm{~s}, 1 \mathrm{H}, \mathrm{CH}), 3.65-3.31\left(\mathrm{~m}, 2 \mathrm{H}, \mathrm{CH}_{2}\right), 2.61-2.14\left(\mathrm{~m}, 2 \mathrm{H}, \mathrm{CH}_{2}\right)$, 1.58-1.39 (m, 3H, $\left.\mathrm{CH}_{3}\right), 0.94\left(\mathrm{~s}, 3 \mathrm{H}, \mathrm{CH}_{3}\right)$; Mass: $m / z=342\left[\mathrm{M}^{+}\right]$; Anal. Calcd for $\mathrm{C}_{15} \mathrm{H}_{17} \mathrm{Cl}_{2} \mathrm{~N}_{3} \mathrm{O}_{2}$ : C, 52.64; H, 5.01; N, 12.28. Found: C, 52.61; H, 4.96; N, 12.28.

$\mathbf{N}$-(4-Nitrophenyl)-4-(4-fluorophenyl)-1,2,3,4-tetrahydro-6-methyl-2-oxopyrimidine-5carboxamide (7). Mp 262-264 C; Yield - 72\%. IR (KBr): 3279, 3244, 3225, 3190, 2852, 1693 , $1674,1614,1529,1448,1357,1240,758 \mathrm{~cm}^{-1} ;{ }^{1} \mathrm{H}$ NMR: $\delta=9.97$ (s, $\left.1 \mathrm{H}, \mathrm{NH}\right), 8.19(\mathrm{~s}, 1 \mathrm{H}, \mathrm{NH})$, $8.16(\mathrm{~s}, 1 \mathrm{H}, \mathrm{NH}), 7.89-6.96(\mathrm{~m}, 8 \mathrm{H}, \mathrm{Ar}), 4.96(\mathrm{~s}, 1 \mathrm{H}, \mathrm{CH}), 1.68\left(\mathrm{~s}, 3 \mathrm{H}, \mathrm{CH}_{3}\right) ; \mathrm{Mass}: m / z=370$ $\left[\mathrm{M}^{+}\right]$; Anal. Calcd for $\mathrm{C}_{18} \mathrm{H}_{15} \mathrm{FN}_{4} \mathrm{O}_{4}: \mathrm{C}, 58.38 ; \mathrm{H}, 4.08 ; \mathrm{N}, 15.13$. Found: C, 58.37; H, 4.04; N, 15.10 .

N-(4-Nitrophenyl)-4-(4-methoxyphenyl)-1,2,3,4-tetrahydro--6-methyl-2-oxopyrimidine-5carboxamide (8). Mp 210-212 ${ }^{\circ} \mathrm{C}$; Yield - 85\%. IR (KBr): 3373, 3248, 3211, 3090, 1689, 1678, 1600, 1529, 1354, 1207, 1186, 840, $758 \mathrm{~cm}^{-1}$; ${ }^{1} \mathrm{H}$ NMR: $\delta=10.21(\mathrm{~s}, 1 \mathrm{H}, \mathrm{NH}), 8.91(\mathrm{~s}, 1 \mathrm{H}, \mathrm{NH})$, $8.54(\mathrm{~s}, 1 \mathrm{H}, \mathrm{NH}), 8.18-6.84(\mathrm{~m}, 8 \mathrm{H}, \mathrm{Ar}), 4.89(\mathrm{~s}, 1 \mathrm{H}, \mathrm{CH}), 3.91\left(\mathrm{~s}, 3 \mathrm{H}, \mathrm{OCH}_{3}\right), 1.74(\mathrm{~s}, 3 \mathrm{H}$, $\left.\mathrm{CH}_{3}\right) ;{ }^{13} \mathrm{C}$ NMR (400 MHz, DMSO) $\delta 14.73\left(\mathrm{CH}_{3}\right), 52.39(\mathrm{C} 4), 58.93\left(\mathrm{OCH}_{3}\right), 108.66(\mathrm{C} 5)$, 
114.39, 114.63, 121.17, 121.54, 127.55, 128.57, 129.35 and 130.06 (CAr.), 131.88 (C-C4, Ar.),

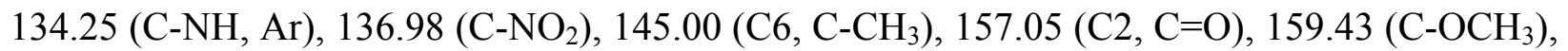
$163.75(\mathrm{CONH})$; Mass: $m / z=382\left[\mathrm{M}^{+}\right]$; Anal. Calcd for $\mathrm{C}_{19} \mathrm{H}_{18} \mathrm{~N}_{4} \mathrm{O}_{5}: \mathrm{C}, 59.68 ; \mathrm{H}, 4.74 ; \mathrm{N}$, 14.65. Found: C, 59.66; H, 4.71; N, 14.63.

$\mathbf{N}$-(4-Nitrophenyl)-4-propyl-1,2,3,4-tetrahydro-6-methyl-2-oxo-pyrimidine-5-carboxamide

(9). Mp 222-224 ${ }^{\circ} \mathrm{C}$; Yield - 95\%. IR (KBr): 3308, 3296, 3277, 2954, 2848, 2822, 1688, 1668, 1545, 1442, 1345, 1207, 1112, $786 \mathrm{~cm}^{-1} ;{ }^{1} \mathrm{H}$ NMR: $\delta=9.03(\mathrm{~s}, 1 \mathrm{H}, \mathrm{NH}), 8.51(\mathrm{~s}, 1 \mathrm{H}, \mathrm{NH}), 8.25$ (s, 1H, NH), 7.38-6.78 (m, 4H, Ar), 5.75 (s, 1H, CH), 3.16-3.13 (m, 2H, CH $), 2.52-2.49$ (m, $\left.2 \mathrm{H}, \mathrm{CH}_{2}\right), 1.98\left(\mathrm{~s}, 3 \mathrm{H}, \mathrm{CH}_{3}\right), 1.77-1.72\left(\mathrm{~m}, 3 \mathrm{H}, \mathrm{CH}_{3}\right)$; Mass: $m / z=318\left[\mathrm{M}^{+}\right]$; Anal. Calcd for $\mathrm{C}_{15} \mathrm{H}_{18} \mathrm{~N}_{4} \mathrm{O}_{4}$ : C, 56.60; H, 5.70; N, 17.60. Found: C, 56.57; H, 5.66; N, 17.57.

\section{Acknowledgements}

Authors are thankful for facilities \& grants given under UGC-SAP for Department Research Support (DRS) and Department of Science \& Technology (DST) New Delhi for Fund for Improvement of Science \& Technology (FIST) and Department of Chemistry for providing laboratory facilities.

\section{References}

1. Kappe, C. O. Tetrahedron 1993, 49, 6937.

2. Rovnyak, G. C.; Atwal, K. S.; Hedberg, A.; Kimball, S. D.; Moreland, S.; Gougoutas, J. Z.; O'Reilly, B. C.; Schwartz, J.; Malley, M. F. J. Med. Chem. 1992, 35, 3254.

3. Khania, E. L.; Sillinietse, G. O.; Ya.Ya. Ozel,; Dabur, G.; Yakimenis, A. A. Khim. Pharm.

Zh. 1978, 12, 1321.

4. Cho, H.; Ueda, M.; Shima, K.; Mizuno, A.; Hayashimatsu, M.; Ohnaka, Y.; Takeuchi, Y.; Hamaguchi, M.; Aisaka, K.; Hidaka, T. J. Med. Chem. 1989, 32, 2399.

5. Varma, R. S. Green Chem. 1999, 1, 43.

6. Rovnyak, G. C.; Kimball, S. D.; Beyer, B.; Cucinotta, G.; DiMarco, J. D.; Gougoutas, J.; Hedberg, A.; Malley, M.; McCarthy, J. P.; Zhang, R. J. Med. Chem. 1995, 38, 119.

7. Loupy, A.; Petit, A.; Hamelin, J.; Texier-Boullet, F.; Jacquault, P.; Mathe, D. Synthesis 1998, 9, 1213.

8. Atwal, K. S.; Swanson, B. N.; Unger, S. E.; Floyd, D. M.; Moreland, S.; Hedberg, A.; O'Reilly, B. C. J. Med. Chem. 1991, 34, 806.

9. Rovnyak, G. C.; Atwal, K. S.; Hedberg, A.; Kimball, S. D.; Moreland, S.; Gougoutas, J. Z.; O'Reilly, B. C.; Schwartz, J.; Malley, M. F. J. Med. Chem. 1992, 35, 3254. 
10. Grover, G. J.; Dzwonczyk, S.; McMullen, D. M.; Normandin, D. E.; Parham, C. S.; Sleph, P. G.; Moreland, S. Cardiovasc. Pharmacol. 1995, 26, 289.

11. Kappe, C. O.; Falsone, F. S. Synlett 1998, 7, 718.

12. Bose, A. K.; Banik, B. K.; Lavlinskaia, N.; Jayaraman, M.; Manhas, M. S. Chemtech 1997, 27, 18.

13. Yarim, M,; Sarac, S.; Ertan, M.; Batu, O.; Erol, K. Farmaco 1999, 54, 359.

14. Foroughifar, N.; Mobinikhaledi, A. Asian J. Chem. 2002, 14, 614.

15. Bruce, M. A.; Poindexter, G. S.; Johnson, G. PCT Int. WO 9833791.Hu, E. H.; Sidler, D. R.; Dolling, U. H. J. Org. Chem. 1998, 63, 3454.

16. Yadav, J. S.; Subba, B. V.; Reddy, S.' Srinivas, R.; Venugopal, C.; Ramalingam, T. Synthesis 2001, 9, 1341.

17. Srinivas, K. V. N. S.; Das, B. Synthesis 2004, 13, 2091.

18. Falsone, F. S.; Kappe, C. O. Arkivoc 2001, (ii), 122.

19. The Merck Index, An Encyclopedia of Chemicals, Drugs and Biologicals, $13^{\text {th }}$ ed., 2001, p 683.

20. Dixon, L. A. In Encyclopedia of Reagents for Organic Synthesis; Paquette, L., Ed.; Wiley: Chichester, 1995; Vol. 6, pp 4166-4169. 